\title{
Application of the Loo-Riegelman Absorption Method
}

\author{
John G. Wagner ${ }^{1}$ \\ Received Feb. 27, 1974 -Final Sept. 4, 1974
}

\begin{abstract}
The Loo-Riegelman absorption method provides the correct $A_{\infty} / V_{1}$ value and the correct rate constant $\mathrm{k}_{\mathrm{a}}$ (if absorption is first order), whether metabolism occurs in compartment $I$ only, compartment 2 only, or both compartments I and 2 of the two-compartment open model. In cases where there is metabolism in compartment 2 , the disposition parameters estimated from intravenous data are only apparent and not the real values. The correct $A_{\infty} / V_{1}$ and $\mathrm{k}_{\mathrm{a}}$ values are obtained, however, only under conditions not hithertofore specified. These conditions are that there must be essentially no bias in the disposition parameters $\mathrm{k}_{12}, \mathrm{k}_{21}$, and $\mathrm{k}_{p 1}$. and in the $\mathrm{C}_{0}$ value estimated from the intravenous data, and that in the oral study a large number of interpolated plasma concentrations, as well as the observed plasma concentrations, must be used, especially for drugs with long half-lives. It is shown that application of the Guggenheim method to the initial $\mathrm{A}_{1}, \mathrm{~V}_{1}, \mathrm{t}$ values frequently provides a better method of estimating $\mathrm{A}_{\infty} / \mathrm{V}_{1}$ and $\mathrm{k}_{\mathrm{a}}$ than the classical method. If biased disposition parameters are used in application of the Loo-Riegelman method to oral data, then essentially the correct value of $\mathrm{k}_{\mathrm{a}}$ will be estimated, but the estimate of $\mathrm{A}_{\infty} / \mathrm{V}_{1}$ will be approximately equal to the true value of $\mathrm{A}_{\infty} / \mathrm{V}_{1}$ multiplied by the ratio of the biased $\mathrm{C}_{0}$ value (obtained in fitting the intravenous data) to the true $\mathrm{C}_{0}$ value of the intravenous data. The above indicates that intravenous data should be fitted by computer until there are no systematic deviations or trends and as small a sum of squared deviations as possible is obtained. The oral data should be fitted by spline or Akima methods, or similar procedures, to produce a function which passes through each observed plasma concentration and at the same time provides a large number of interpolated concentration data.
\end{abstract}

KEY WORDS: absorption plot; a mount absorbed; kinetics of absorption; bias in computer fitting: disposition parameters; interpolation of blood levels.

\section{INTRODUCTION}

Loo and Riegelman (1) derived equations 1 and 2:

$$
A_{t_{n}} / V_{1}=\left(C_{1}\right)_{t_{n}}+k_{\mathrm{el}} \int_{t_{0}}^{t_{n}} C_{1} d t+\left(C_{2}\right)_{t_{n}}
$$

\footnotetext{
Partly supported by Public Health Service Grant 5-P11-GM15559.

${ }^{1}$ College of Pharmacy and Upjohn Center for Clinical Pharmacology, The University of Michigan, Ann Arbor. Michigan 48104.
} 


$$
\begin{aligned}
\left(C_{2}\right)_{t_{n}}= & \left(C_{2}\right)_{t_{n-1}} \cdot e^{-k_{21} \Delta t}+k_{12} / k_{21} \cdot\left(C_{1}\right)_{t_{n-1}}\left(1-e^{-k_{21} \Delta t}\right) \\
& +k_{12} \Delta C_{1} \Delta t / 2
\end{aligned}
$$

Although not explicitly stated in their article (1), it is implicit that the asymptote of a plot of $A_{t_{n}} / V_{1}$ is $A_{\propto} / V_{1}$, which is given by equation 3 :

$$
A_{\infty} / V_{1}=k_{\mathrm{e} 1} \int_{t_{0}}^{\infty} C_{1} d t
$$

In equations $1-3, A_{t_{n}} / V_{1}$ is the cumulative amount of drug absorbed to time $t_{n}$ divided by the volume of the inner (central) compartment of Model II below (if drug is given by intravenous infusion it is the amount which has been infused to time $t_{n}$ divided by $\left.V_{1}\right),\left(C_{1}\right)_{t_{n}}$ is the concentration of drug in the central compartment (assumed to be the equivalent of the plasma concentration), $\left(C_{2}\right)_{t_{n}}$ is the amount of drug in the outer so-called tissue compartment at time $t_{n}$ divided by the volume of the inner compartment (i.e., $A_{2} / V_{1}$ ), the integral is the area under the $C_{1}, t$ curve from time $t_{0}$ (when absorption commences) to time $t_{n}$, and the rate constants $k_{12}, k_{21}$, and $k_{\mathrm{e}}$ refer to those of Model II below. In equation 3, $A_{\infty} / V_{1}$ represents the total amount of drug absorbed divided by the volume $V_{1}$. Usually $t_{0}$ is taken equal to zero since the integral is closely approximated by the trapezoidal rule, and, in such a case, one assumes $\left(C_{1}\right)=0$ when $t=0$, where the latter is the time of dosing.

Loo and Riegelman (1) showed that the $A_{t_{n}} / V_{1}, t_{n}$ data resulting from application of equations 1 and 2 could be analyzed to obtain the kinetics of input of drug to the bloodstream. This was shown by infusing an aspirin solution intravenously in man with a logarithmic infusion pump and backcalculating the first-order infusion rate. They also infused griseofulvin at a constant rate and accurately back-calculated the infusion rate. Subsequently, they reported (2) that when the interval between blood samples became too long the linear piecewise integration procedure used to estimate $\left(C_{2}\right)_{t_{n}}$ resulted in poor estimates, and they proposed a new equation which was based on a logarithmic piecewise procedure which they claimed solved the problem. Another variation in which the value of $k_{\mathrm{el}}$ could be adjusted from the intravenous and oral experiments was reported (3).

The Loo-Riegelman method (1-3) is based on Model II below, but in a given practical situation one does not know whether the true model is Model I, Model II, or Model III. Recently, Suzuki and Saitoh (4) showed that the result obtained by application of the Loo-Riegelman method was independent of the ratio of $k_{\mathrm{el}_{1}} / k_{\mathrm{el}_{2}}$ if the true model was Model I. Kaplan (5) showed that for data collected on coumermycin $A_{1}$ the absorption plot obtained by the Loo-Riegelman method, based on Model II, and the absorption plot, based on Model III, were identical, but they did not give 

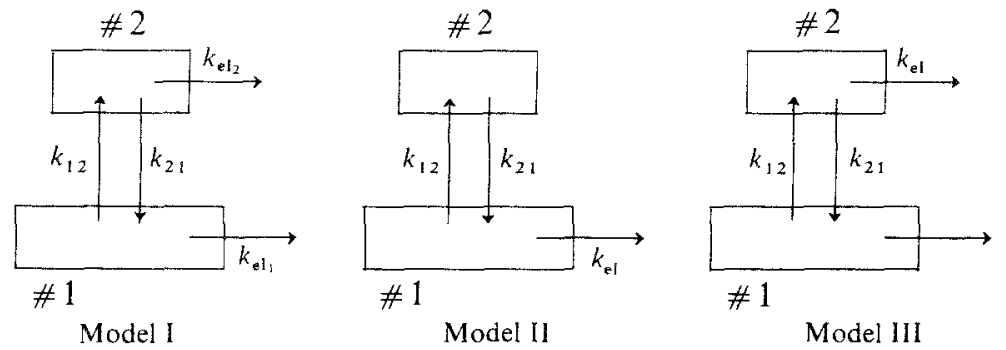

the reason for the result. Analogously, Breckenridge and Orme (6) applied both the Loo-Riegelman method, based on Model II, and the corresponding equations of Kaplan (5), based on Model III, to intravenous and oral warfarin plasma concentration data. They reported that the absorption plots obtained by the two methods were identical, but did not give the reason for the result. This report will show why these authors obtained the results that they reported.

In addition, the effect of bias on the estimates of $k_{12}, k_{21}$, and $k_{\mathrm{el}}$ obtained from intravenous data on the result obtained by application of the Loo-Riegelman method to plasma concentrations observed in the same subject following oral administration has not been reported. Bias in such parameter estimates may exist either from (1) computer fitting of intravenous data using initial estimates of the parameters which are too distant from the real values, resulting in convergence at a local minimum, or (2) using graphical estimates of parameters, as has been shown by Wagner (7) and Wagner and Metzler (8).

Since the Loo-Riegelman method assumes that the plasma concentration curve is linear between two adjacent points, then the interval or intervals between concentrations measured become very critical in the result attained with the method. This problem is examined further, and some suggestions are made to eliminate this problem as a major source of error.

\section{EXPERIMENTAL AND RESULTS}

\section{Effect of Metabolism in Compartment 2}

Define: $A_{T}=$ amount of drug absorbed to time $T$ (same as $A_{i_{n}}$ in equation 1).

$C_{2}=A_{2} / V_{1}=$ amount of drug in compartment 2 at time $t /$ volume of compartment No. 1 .

For Model I,

$$
A_{T} / V_{1}=\left(C_{1}\right)_{T}+\left(C_{2}\right)_{T}+k_{\mathrm{e} 1_{1}} \int_{0}^{T} C_{1}(t) d t+k_{\mathrm{e} 1_{2}} \int_{0}^{T} C_{2}(t) d t
$$




$$
A_{x} / V_{1}=k_{\mathrm{e} l_{1}} \int_{0}^{x} C_{1}(t) d t+k_{\mathrm{el}_{2}} \int_{0}^{x} C_{2}(t) d t
$$

Since there is no way of knowing $k_{\mathrm{el}_{2}}$ unless drug is measured in compartment 2 as well as in compartment 1, then one cannot apply these equations.

Model II is assumed in applying the method of Loo and Riegelman (1):

$$
A_{T} / V_{1}=\left(C_{1}\right)_{T}+\left(C_{2}\right)_{T}+k_{\mathrm{e} 1} \int_{0}^{T} C_{1}(t) d t
$$

If the model is really Model II (i.e., elimination occurs only from compartment 1), then the Loo-Riegelman method provides the correct asymptote $\left(A_{x} / V_{1}\right)$, the correct $A_{T} / V_{1}$ plot, and the correct values of $k_{12}, k_{21}$, and $k_{\mathrm{e} 1}$. If the model is really Model I and one applies Model II in the form of the Loo-Riegelman method, the method gives the correct asymptote $\left(A_{\propto} / V_{1}\right)$, the correct $A_{T} / V_{1}$ vs. $T$ plot (i.e., the correct kinetics of absorption are obtained), but only apparent values, $\left(k_{12}\right)_{\mathrm{app}},\left(k_{21}\right)_{\mathrm{app}}$, and $\left(k_{\mathrm{el}}\right)_{\mathrm{app}}$, of the disposition portion of the model are obtained, and not the real values, $k_{12}$, $k_{21}$, and $k_{\mathrm{el}_{1}}$. The reason for this is as follows.

When drug is administered intravenously as a bolus of dose $D$, the plasma concentration-time curve is described by the equation

$$
C_{1}(t)=A e^{-\alpha t}+B e^{-\beta t}
$$

Formulas used to obtain estimates of microscopic rate constants give the following results:

$$
\begin{gathered}
\left(k_{21}\right)_{\mathrm{app}}=\left\{A \beta+B \alpha_{\}}\right\}\{A+B\} \\
\left(k_{\mathrm{el}}\right)_{\mathrm{app}}=\alpha \beta /\left(k_{2 \mathrm{l}}\right)_{\mathrm{app}}=\left\{k_{21} \dot{k}_{\mathrm{el}_{1}}+k_{12} k_{\mathrm{e}_{2}}+k_{\mathrm{el}_{1}{ }_{\mathrm{e} l_{2}}}\right\} /\left\{k_{21}+k_{\mathrm{el}_{2}}\right\}
\end{gathered}
$$

Hence

$$
\begin{aligned}
A_{x} / V_{1}= & \left(k_{\mathrm{e} 1}\right)_{\mathrm{app}} \int_{0}^{\infty} C_{1}(t) d t \\
& =\left[\left\{k_{21} k_{\mathrm{e}_{1}}+k_{12} k_{\mathrm{el}_{2}}+k_{\mathrm{el}_{1}} k_{\mathrm{el}_{2}}\right\} /\left\{k_{21}+k_{\mathrm{el}_{2}}\right\}\right] \int_{0}^{\infty} C_{1}(t) d t
\end{aligned}
$$

For Model I, working in amounts $(A)$

$$
A_{1}=D\left[\left(E_{2}-\beta\right) e^{-\beta t}-\left(E_{2}-\alpha\right) e^{-\alpha t}\right] /(\alpha-\beta)
$$

where

$$
\begin{gathered}
E_{2}=k_{21}+k_{\mathrm{e} 1_{2}} \\
\int_{0}^{x} A_{1}(t) d t=E_{2} D / \alpha \beta \\
A_{2}=k_{12} D\left[e^{-\beta t}-e^{-x t}\right] /(\alpha-\beta)
\end{gathered}
$$




$$
\begin{aligned}
\int_{0}^{\infty} A_{2}(t) d t & =k_{12} D / \alpha \beta \\
\int_{0}^{\infty} A_{2}(t) d t / \int_{0}^{\infty} A_{1}(t) d t & =\int_{0}^{\infty} C_{2}(t) d t / \int_{0}^{\infty} C_{1}(t) d t \\
& =k_{12} / E_{2}=k_{12} /\left\{k_{21}+k_{\mathrm{e}_{2}}\right\}
\end{aligned}
$$

And

$$
\int_{0}^{\infty} C_{2}(t) d t=\left\{k_{12} /\left(k_{21}+k_{\mathrm{e}_{2}}\right)\right\} \int_{0}^{\infty} C_{1}(t) d t
$$

Substituting from equation 17 into equation 5 gives

$$
\begin{aligned}
& A_{x_{1}} / V_{1}=\left[k_{\mathrm{el}_{1}}+\left\{k_{12} k_{\mathrm{el}_{2}} /\left(k_{21}+k_{\mathrm{el}_{2}}\right)\right\}\right] \int_{0}^{\infty} C_{1}(t) d t \\
& =\left[\left\{k_{21} k_{\mathrm{el}_{1}}+k_{12}+k_{\mathrm{el}_{2}}+k_{\mathrm{el}_{1}{ }_{1} k_{2}}\right\} /\left\{k_{21}+k_{\mathrm{el}_{2}}\right\}\right] \int_{0}^{\infty} C_{1}(t) d t
\end{aligned}
$$

Since the right-hand sides of equations 10 and 18 are the same, the LooRiegelman method gives the correct amount absorbed even if the model is Model I. Simulations have shown that it also gives the correct kinetics of absorption. An example is given below.

Simulation Example 1. For the intravenous data, let $k_{12}=0.37, k_{21}=$ $0.23, k_{\mathrm{el}_{1}}=0.08, k_{\mathrm{el}_{2}}=0.08, C_{0}=D / V_{1}=25$.

For Model I, we have

$$
\begin{aligned}
& C_{1}(t)=C_{0}\left[\left(k_{21}+k_{\mathrm{e} 1}-\beta\right) e^{-\beta t}-\left(k_{21}+k_{\mathrm{el}_{2}}-\alpha\right) e^{-x t}\right] /(\alpha-\beta) \\
& C_{1}(t)=A e^{-x t}+B e^{-\beta t} \\
& C_{1}(t)=15.417 e^{-0.68 t}+9.583 e^{-0.08 t}
\end{aligned}
$$

For Model II, we calculate

$$
\begin{aligned}
\left(k_{21}\right)_{\mathrm{app}}= & (A \beta+B \alpha) /(A+B)=k_{21}+k_{\mathrm{el}_{2}}=0.31 \\
\left(k_{\mathrm{el}}\right)_{\mathrm{app}}= & \alpha \beta /\left(k_{21}\right)_{\mathrm{app}}=(0.68)(0.08) / 0.31=0.1755 \\
\left(k_{12}\right)_{\mathrm{app}}= & \alpha+\beta-\left(k_{21}\right)_{\mathrm{app}}-\left(k_{\mathrm{el}}\right)_{\mathrm{app}}=0.68+0.08 \\
& -0.31-0.1755=0.2745
\end{aligned}
$$

Note that

$$
\int_{0}^{\infty} C_{1}(t) d t=(15.417 / 0.68)+(9.583 / 0.08)=142.5
$$


and

$$
\left(k_{\mathrm{el}}\right)_{\mathrm{app}} \int_{0}^{\infty} C_{1}(t) d t=(0.1755)(142.5)=25 .
$$

In the above,

$$
\begin{aligned}
\alpha+\beta & =k_{12}+k_{21}+k_{\mathrm{el}_{1}}+k_{\mathrm{el}_{2}}=0.76 \\
\alpha \beta & =k_{21} k_{\mathrm{el}_{1}}+k_{12} k_{\mathrm{el}_{2}}+k_{\mathrm{el}_{1}} k_{\mathrm{el}_{2}}=E_{1} E_{2}-k_{12} k_{21}=0.0544
\end{aligned}
$$

where $E_{1}=k_{12}+k_{\mathrm{el}_{1}}$ and $E_{2}=k_{21}+k_{\mathrm{el}_{2}}$

$$
\begin{aligned}
& \alpha=\frac{1}{2}\left[(\alpha+\beta)+\sqrt{(\alpha+\beta)^{2}-4 \alpha \beta}\right]=0.68 \\
& \beta=\frac{1}{2}\left[(\alpha+\beta)-\sqrt{(\alpha+\beta)^{2}-4 \alpha \beta}\right]=0.08
\end{aligned}
$$

Oral data were generated with the appropriate equation for Model I, namely

$$
\begin{aligned}
C_{1}(t)= & k_{a} C_{0}\left[\left\{\left(E_{2}-\alpha\right) /\left(k_{a}-\alpha\right)(\beta-\alpha)\right\} e^{-\alpha t}+\left\{\left(E_{2}-\beta\right) /\left(k_{a}-\beta\right)(\alpha-\beta) e^{-\beta 1}\right.\right. \\
& \left.+\left\{\left(E_{2}-k_{a}\right) /\left(\alpha-k_{a}\right)\left(\beta-k_{a}\right)\right\} e^{-k_{a} t}\right] \\
C_{1}(t)= & A_{1} e^{-\alpha t}+A_{2} e^{-\beta t}+A_{3} e^{-k_{a} t} \\
C_{1}(t)= & -23.4105 e^{-0.68 t}+11.9066 e^{-0.08 t}+11.5039 e^{-0.41 t}
\end{aligned}
$$

\begin{tabular}{|c|c|c|c|c|c|c|c|}
\hline \multirow{2}{*}{$t_{n}$} & \multirow{2}{*}{$\left(C_{1}\right)_{2_{n}}$} & \multirow{2}{*}{$\int_{0}^{t_{n}} C_{1}(t) d t$} & \multirow{2}{*}{$\left(k_{\mathrm{el}}\right)_{\mathrm{app}}$} & \multirow{2}{*}{$\left(\hat{C}_{2}\right) t_{n}^{a}$} & \multirow{2}{*}{$A_{t_{n}} / V_{1}^{b}$} & \multicolumn{2}{|c|}{ Guggenheim data } \\
\hline & & & & & & & $\Delta\left(A_{i_{n}} / V_{1}\right)$ \\
\hline 0 & 0 & 0 & 0 & 0 & 0 & 0 & \\
\hline 0.5 & 4.1485 & 1.0371 & 0.1820 & 0.2847 & 4.6152 & & 4.6152 \\
\hline 1.0 & 6.7656 & 3.7657 & 0.6609 & 0.9509 & 8.3774 & 0.5 & $\begin{array}{l}3.7622 \\
3.0666\end{array}$ \\
\hline 1.5 & 8.3380 & 7.5416 & 1.3236 & 1.7824 & $11.4440^{-}$ & -1.0 & 3.0666 \\
\hline 2.0 & 9.2042 & 11.9271 & 2.0932 & 2.6461 & 13.9435 & 1.5 & 2.4995 \\
\hline 2.5 & 9.5991 & 16.6279 & 2.9182 & 3.4635 & 15.9808 & $>2.0$ & 2.0373 \\
\hline 3.0 & 9.6845 & 21.4488 & 3.7643 & 4.1925 & 17.6413 & $>2.5$ & 1.6605 \\
\hline 3.5 & 9.5714 & 26.2628 & 4.6091 & 4.8140 & 18.9945 & $>3.0$ & 1.3532 \\
\hline 4.0 & 9.3353 & 30.9895 & 5.4387 & 5.3235 & $20.0975^{-}$ & .5 & 1.1030 \\
\hline 5.0 & 8.6809 & 39.9976 & 7.0196 & 6.0181 & 21.7186 & & \\
\hline 6.0 & 7.9546 & 48.3153 & 8.4793 & 6.3632 & 22.7971 & & \\
\hline 7.0 & 7.2529 & 55.9191 & 9.8138 & 6.4483 & 23.5150 & & \\
\hline 9.0 & 6.0314 & 69.2034 & 12.1452 & 6.1010 & 24.2776 & & \\
\hline 11.0 & 5.0520 & 80.2868 & 14.0903 & 5.4809 & 24.6231 & & \\
\hline 15.0 & 3.6099 & 97.6106 & 17.1307 & 3.9733 & $24.7139)$ & & \\
\hline 18.0 & 2.8281 & 107.2676 & 18.8255 & 3.1811 & $24.8347\}$ & Ave & rage $=24.7491$ \\
\hline 24.0 & 1.7462 & 120.9905 & 21.2338 & 1.7187 & $24.6987)$ & & \\
\hline
\end{tabular}

Table I. Simulation Example 1 Showing That Loo-Riegelman Method Gives Correct Result if the Model Is Model I

${ }^{a}$ Calculated with equation 2 .

${ }^{b}$ Calculated with equation 1 . 
where $A_{1}=-23.4105, \alpha=0.68, A_{2}=11.9066, \beta=0.08, A_{3}=11.5039$, and $k_{a}=0.41$. Data generated are shown in columns 1 and 2 of Table I. The Loo-Riegelman method was applied to these data using the values as follows: $\left(k_{12}\right)_{\mathrm{app}}=0.2745,\left(k_{21}\right)_{\mathrm{app}}=0.31,\left(k_{\mathrm{el}}\right)_{\mathrm{app}}=0.1755$. Results are shown in columns 5 and 6 of Table $\mathrm{I}$.

Application of the Guggenheim Method to Estimate $k_{a}$

The data for $k_{a}$ according to the Guggenheim method are shown in the last two columns of Table I. Linear least-squares regression of $\ln \left\{\Delta\left(A_{t_{n}} / V_{1}\right)\right\}, t_{1}$ values gave the equation

$$
\ln \left[\Delta\left(A_{t_{n}} / V_{1}\right)\right]=1.5295-0.409 t_{1}
$$

whence

$$
\Delta\left(A_{t_{n}} / V_{1}\right)=4.6158 e^{-0.409 i_{1}}
$$

Hence $\hat{k}_{a}$ is $0.409 \mathrm{hr}^{-1}$ and the real value was 0.41 , and

$$
\begin{aligned}
\hat{C}_{0}=A_{x} / V_{1} & =4.6158 /\left\{1-e^{-\left(k_{a}\right)(\Delta t)}\right\}=4.6158 /\left\{1-e^{-(0.409)(0.50)}\right\} \\
& =24.96
\end{aligned}
$$

whereas the real value is 25 .

Hence if the estimates are rounded off the estimates are equal to the real values.

Usual Methods of Estimating $k_{a}$ and $C_{0}$

Usually the estimate of $A_{x} / V_{1}$ is taken from the terminal values of $A_{t_{n}} / V_{1}$, i.e., the asymptote of the $A_{t_{n}} / V_{1}$ vs. $t_{n}$ plot. If we take the average value of the three last $A_{t_{n}} / V_{1}$ values, namely 24.7491 , as the $A_{\infty} / V_{1}$ value and then do linear least-squares regression on $\ln \left(24.7491-A_{t_{n}} / V_{1}\right), t_{n}$ values, we obtain

$$
\ln \left(24.7491-A_{t_{n}} / V_{1}\right)=3.2337-0.428 t(r=0.9999)
$$

whence

$$
\left\{24.7491-A_{t_{n}} / V_{1}\right\}=25.375 e^{-0.428 t}
$$

Hence using this method we have 0.428 as the estimate of $k_{a}$ and two estimates of $A_{\infty} / V_{1}$, namely 24.7491 and 25.375 , and we really don't know which one is the "correct" one.

Note that the Guggenheim method of estimating $k_{a}$ and $C_{0}$ or $A_{\infty} / V_{1}$ is the more accurate in this case and is less ambiguous.

The product of $\left(k_{e l}\right)_{\text {app }}$ and the total area under the "plasma concentration" curve is also an estimate of $A_{\infty} / V_{1}$. With the example given, the result 
is shown below:

$$
\begin{aligned}
\int_{0}^{\infty} C_{1}(t) d t & =A_{1} / \alpha+A_{2} / \beta+A_{3} / k_{a} \\
& =-23.4105 / 0.68+11.9066 / 0.08+11.5039 / 0.41 \\
& =142.5 \\
A_{x} / V_{1} & =\left(k_{\mathrm{el}}\right)_{\mathrm{app}} \int_{0}^{\infty} C_{1}(t) d t=(0.1755)(142.5)=25
\end{aligned}
$$

\section{Effect of Bias in Parameters Estimated from Intravenous Data}

Simulation Example 2. For the intravenous data, the parameter values $k_{12}=1.162, k_{21}=0.515, k_{\mathrm{e} 1}=0.038$, and $C_{0}=100$ (corresponding to $V_{1}=41.0$ ) were used to substitute into equation 41 , which is appropriate for Model II. Such substitution gave equation 42 :

$$
\begin{aligned}
& C_{1}(t)=C_{0}\left[\left(k_{21}-\beta\right) e^{-\beta t}-\left(k_{21}-\alpha\right) e^{-x t}\right] /(\alpha-\beta) \\
& C_{1}(t)=29.758 e^{-0.0115 t}+70.2419 e^{-1.7035 t}
\end{aligned}
$$

Intravenous $C_{1}, t$ values were generated with equation 42 and 20 sets of concentrations "with noise" were generated by adding $5 \%$ random error with normal deviates. Each of these 20 sets of concentration data was fitted to equation 41 using the program NONLIN and an IBM digital computer. The initial estimates used to initiate the iteration in each case were values of $k_{12}, k_{21}, k_{\mathrm{el}}$, and $V_{1}$ which were twice the known values. The averages of the 20 estimated parameters of each type were $k_{12}=1.853, k_{21}=0.797, k_{\mathrm{el}}=$ 0.068 , and $V_{1}=32.6$ (corresponding to $C_{0}=125.8$ ). The real values of $\alpha$ and $\beta$ were $\alpha=1.7035$ and $\beta=0.0155$. The values of $\alpha$ and $\beta$ obtained from the averaged parameters were $\alpha=2.6979$ and $\beta=0.0201$. The averages of the parameters, obtained by this method, are quite biased and represent an extreme case. We could have used only one of 20 sets of biased estimates but reasoned that it was fairer to use the averages of the 20 . The equation for the estimated concentrations $\left(C_{1}\right)$, obtained by substituting the averaged parameters into equation 41 . is given by equation 43 :

$$
\widehat{C}_{1}(t)=36.489 e^{-0.0201 t}+89.2805 e^{-2.6979 t}
$$

A table of the $\hat{C}_{1}$ and real $C_{1}$ values clearly shows the bias in that when $t<12$ then $\hat{C}_{1}>C_{1}$, and when $t>24, \hat{C}<C_{1}$. However, because of the rapid falloff and then flattening of the curve, it is almost impossible to show the nature of the systemic deviations or trends on cartesian roordinate graph 

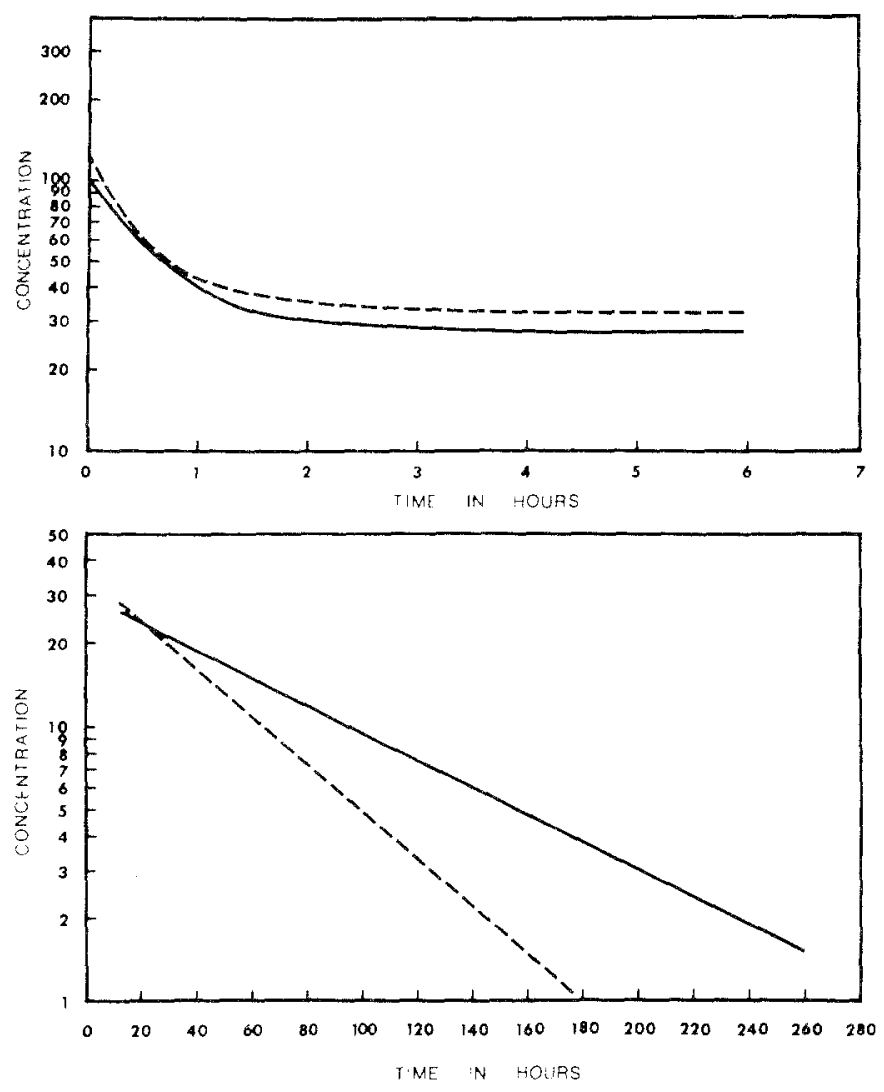

Fig. 1. Simulated intravenous blood level curve (- - ) generated with equation 42 and biased estimations (- - -) generated with equation 43. Top: Expanded scale of first 6 hr only. Bottom: Terminal part of curves.

paper. Figure 1 consists of two semilogarithmic plots which show the nature of the trends. At the top of Fig. 1 is an expanded scale plot showing the data for the first $6 \mathrm{hr}$. At the bottom of Fig. 1 some of the data beyond $10 \mathrm{hr}$ are plotted. A weighted residual plot would also show such systematic deviations.

Oral $C_{1}, t$ data were generated by using the same real values of the disposition parameters as used to generate the original intravenous data. Three sets were generated using $C_{0}=100$ and $k_{a}=0.5, C_{0}=75$ and $k_{a}=1.25$, and $C_{0}=75$ and $k_{a}=2$. The parameter values were substituted into equation 31 and the value of $k_{21}=0.515$ was used for $E_{2}$. The LooRiegelman method was then applied to the $C_{1}, t$ data of each of the three sets using the biased parameters $k_{12}=1.853, k_{21}=0.797$, and $k_{\mathrm{e} 1}=0.068$. In each set, concentrations were used at times $t=0.2,0.4 .0 .6,0.8 .1 .0 .1 .2 .1 .4$, 
Table II. Estimates of $A_{x} / V_{1}$ and $k_{a}$ Obtained by Using Biased Disposition Parameters in Applying the Loo-Riegelman Method in Simulation Example 2

\begin{tabular}{|c|c|c|c|c|c|c|}
\hline & \multicolumn{2}{|c|}{ Set 1} & \multicolumn{2}{|c|}{ Set 2} & \multicolumn{2}{|c|}{ Set 3} \\
\hline & $A_{\infty} / V_{1}$ & $k_{a}$ & $A_{\infty} / V_{1}$ & $k_{a}$ & $A_{\infty} / V_{1}$ & $k_{a}$ \\
\hline Real value & 100 & 0.50 & 75 & 1.25 & 75 & 2.00 \\
\hline Guggenheim method & 123 & 0.48 & 108 & 0.91 & 96.4 & 1.64 \\
\hline Sigma-minus method & 119 & 0.53 & 99.5 & 1.48 & 101.0 & 2.26 \\
\hline$\left(\text { real } \frac{A_{\propto}}{V_{1}}\right)_{\text {oral }} \times \frac{\left(\text { biased } C_{0}\right)_{\text {i.v. }}}{\left(\text { real } C_{0}\right)_{\text {i.v. }}}$ & 125.8 & - & 94.4 & - & 94.4 & \\
\hline
\end{tabular}

$1.6,1.8,2,4,6,8,10,12,15,18,21,24,36,48,60,72,84,96,120,144,168,192$. $216,240,264,288,312$. These are more sampling times than one would have available in a human study, but we wished not to introduce another source of error discussed later.

The Guggenheim method was applied to the equally spaced $\Delta A_{T} / V_{1}, t_{1}$ values (here $t_{1}$ is time at the beginning of the interval) in the 0 - to 2-hr range, and the sigma-minus method was also applied to estimate $A_{\infty} / V_{1}$ and $k_{a}$ from each set of data. Results are shown in Table II. It may be seen that $k_{a}$ is reasonably well estimated in each case but that $A_{\infty} / V_{1}$ is always appreciably higher than the real value. This is due to the bias in the fitting of the intravenous data. The ratio of volumes is $41 / 32.6=1.258$, hence the ratio

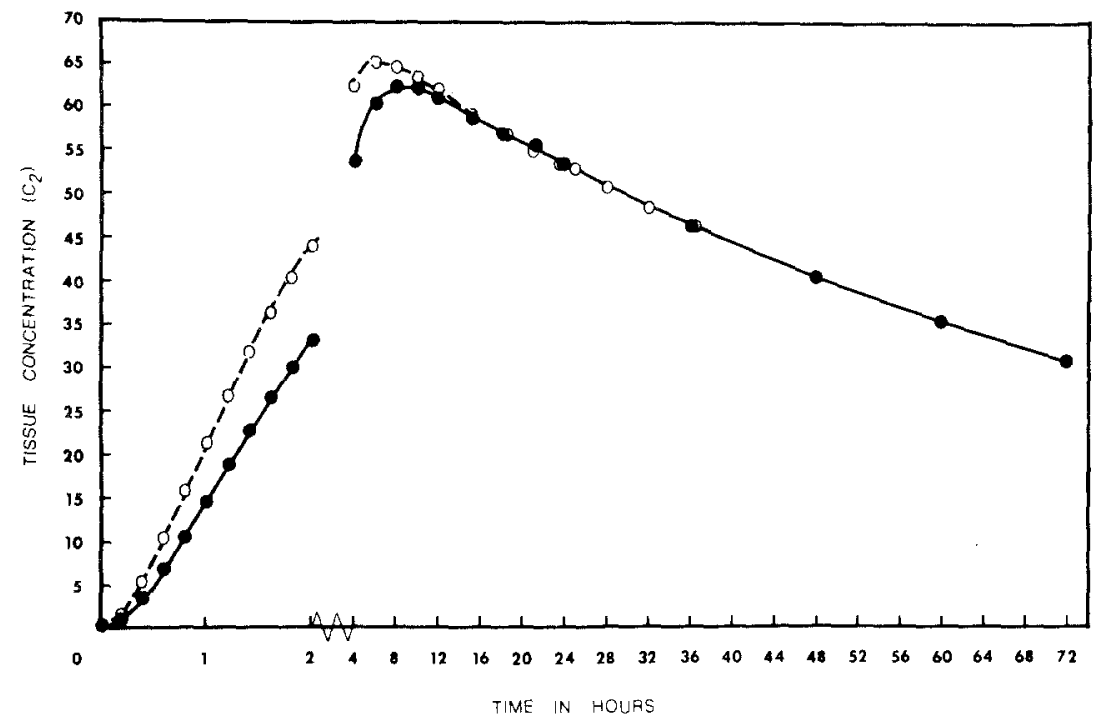

Fig. 2. Solid line gives actual $C_{2}$ values for simulation example 2 (oral). Dotted line gives estimated $C_{2}$ values calculated with the biased parameter values. 
of biased $C_{0} /$ true $C_{0}=125.8 / 100=1.258$. Equation 44 approximately holds in each case:

$$
\left(A_{\infty} \hat{\infty} / V_{1}\right)_{\text {oral }} \simeq\left(\text { real } A_{\infty} / V_{1}\right)_{\text {oral }} \cdot\left(\text { biased } C_{0}\right)_{\text {i.v. }} /\left(\text { real } C_{0}\right)_{\text {i.v. }}
$$

That is, the poor estimate of $A_{\infty} / V_{1}$ obtained by application of the LooRiegelman method to the oral data is accounted for by the bias in the estimate of $C_{0}$ in the fitting of the intravenous data. This can be seen by comparing the values of the right-hand side of equation 44 with the $A_{\infty} / V_{1}$ estimates in Table II. It is somewhat remarkable that $k_{a}$ is estimated as well as it is under conditions of such biased estimates. This is accentuated by Fig. 2, which is a plot of the real $C_{2}$ values and the values of $\vec{C}_{2}$ obtained by the Loo-Riegelman method using the biased estimates of $k_{12}, k_{21}$, and $k_{\mathrm{e}}$ for set 1 where $k_{a}=0.5$. It is obvious that the $\hat{C}_{2}$ values during the absorption phase are considerably higher in this case than the real $C_{2}$ values.

\section{Effects of Time Interval Between Plasma Concentrations}

Many simulations have been performed, but results of only one will be given.

Simulation Example 3. The original parameters of example 2, namely $k_{12}=1.162, k_{21}=0.515, k_{\mathrm{el}}=0.038, k_{a}=0.5$, and $C_{0}=100$, were used. These values, substituted into equation 31 , gave the equation for $C_{1}$ shown in the footnote to Table III. Substitution into the appropriate equation for Model II also give the equation for $C_{2}$ shown in the same footnote. Using equation $2, \hat{C}_{2}$ values were calculated from the $C_{1}, t$ values listed in columns 1 and 2 of Table III ; in this case, the real values of $k_{12}, k_{21}$, and $k_{\mathrm{e} 1}$ (above) were used and not the biased estimates. Hence this simulation is a test of the criticality of the values of $\Delta C_{1}$ and $\Delta t$ and of the distribution of the $C_{1}$ values with respect to time. Four different sets of $C_{1}, t$ values were used and the generated $\hat{C}_{2}$ values are shown in Table III in the last four columns. All calculations were carried out to the number of significant places shown in Table III. Although the trapezoidal areas are not listed, the error introduced by use of the trapezoidal rule in this simulation was of minor importance in determining the results. Since $\Delta t$ appears in the exponent of " $e$ " in two terms of equation 1, and both $\Delta C_{1}$ and $\Delta t$ appear in the third term, the value of $\Delta t$ and its change with time are the major source of poor estimates of $C_{2}$. When a very large number of $C_{1}, t$ values are used, as in set $1, \hat{C}_{2}$ is essentially the same as the real $C_{2}$ over the whole time range. However, in sets 2 and 3 , when $\Delta t$ suddenly jumps from $2 \mathrm{hr}$ (in the 2- to 12-hr range) to $12 \mathrm{hr}$ (between 12 and $24 \mathrm{hr}$ ) then the $\hat{C}_{2}$ estimated at $24 \mathrm{hr}$ is appreciably lower than the real $C_{2}$ value. At least with this set of data, when fewer "blood samples" are taken in the absorption phase (sets 3 and 4 compared with set 
Table III. How "Tissue Concentration" $\left(C_{2}\right)$ Estimated by the Loo- Riegelman Method Depends Markedly on the Values of $\Delta C_{1}$ and $\Delta t$ (Simulation Example 3)

\begin{tabular}{|c|c|c|c|c|c|c|}
\hline \multirow{2}{*}{$\begin{array}{l}\text { Time } \\
\text { (hr) }\end{array}$} & \multicolumn{2}{|c|}{ Actual values ${ }^{a}$} & \multicolumn{4}{|c|}{$\hat{C}_{2}$ estimated by Loo-Riegelman method } \\
\hline & $C_{1}$ & $C_{2}$ & Set 1 & Set 2 & Set 3 & Set 4 \\
\hline 0 & 0 & 0 & 0 & 0 & 0 & 0 \\
\hline 0.2 & 8.4772 & 1.0050 & 0.9851 & 0.9851 & & - - \\
\hline 0.4 & 14.5103 & 3.4940 & 3.4617 & 3.4617 & 3.3722 & $\cdots$ \\
\hline 0.6 & 18.8027 & 6.8653 & 6.8260 & 6.8260 & & 6.5546 \\
\hline 0.8 & 21.8551 & 10.7075 & 10.6649 & 10.6649 & 10.5464 & \\
\hline 1.0 & 24.0237 & 14.7430 & 14.6994 & 14.6994 & - & -- \\
\hline 1.2 & 25.5620 & 18.7878 & 18.7446 & 18.7446 & 18.6247 & 18.4459 \\
\hline 1.4 & 26.6506 & 22.7231 & 22.6815 & 22.6815 & & - \\
\hline 1.6 & 27.4180 & 26.4756 & 26.4360 & 26.4360 & 26.3261 & -- \\
\hline 1.8 & 27.9558 & 30.0031 & 29.9659 & 29.9659 & $\ldots$ & 29.7084 \\
\hline 2. & 28.3292 & 33.2846 & 33.2500 & 33.2500 & 33.1537 & - \\
\hline-4 & 28.8842 & 53.7895 & 53.6152 & 53.6152 & 53.5808 & 53.5108 \\
\hline 6. & 28.3629 & 60.6871 & 60.4402 & 60.4402 & 60.4279 & $\ldots$ \\
\hline 8. & 27.7577 & 62.3045 & 62.0230 & 62.0230 & 62.0186 & 61.0682 \\
\hline 10 & 27.1408 & 61.9908 & 61.6965 & 61.6965 & 61.6949 & -- \\
\hline 12. & 26.5289 & 60.9871 & 60.6907 & 60.6907 & 60.6901 & 59.5752 \\
\hline 15. & 25.6317 & 59.1009 & 58.4714 & $-\ldots$ & $-\cdot$ & \\
\hline 18. & 24.7630 & 57.1373 & 56.2262 &.- & $\ldots$ & - \\
\hline 21. & 23.9233 & 55.2088 & 54.4844 & -- & - & $\cdots$ \\
\hline 24. & 23.1121 & 53.3386 & 52.6725 & 36.0372 & 36.0372 & 36.0349 \\
\hline 27. & 21.8109 & 51.5302 & 50.0094 & -- & & $-\cdots$ \\
\hline 30. & 21.0815 & 49.7828 & 48.1112 & - & & - \\
\hline 33. & 20.3673 & 48.0946 & 46.4378 & $-\cdots$ & . & $-\cdot$ \\
\hline 36. & 20.1329 & 46.4637 & 45.6494 & 31.3437 &.- & 31.3437 \\
\hline 39. & 19.4502 & 44.8880 & 44.2838 & - & $\ldots$ & - \\
\hline 42. & 18.7906 & 43.3658 & 42.8209 & $\ldots$. &.- & - . \\
\hline 45 & 18.1534 & 41.8952 & 41.3771 & $-\cdots$ & - & $-\cdots$ \\
\hline 48 & 17.5377 & 40.4745 & 39.9756 & 27.3032 & -23.5814 & 30.5258 \\
\hline 51. & 16.9430 & 39.1019 & 38.6204 &.-- & $-\cdots$ & - \\
\hline 54. & 16.3684 & 37.7759 & 37.3107 & -- & - & - \\
\hline 57. & 15.8134 & 36.4945 & 36.0456 & & - & - \\
\hline 60. & 15.2771 & 35.2572 & 34.8232 & 23.7842 & $-\cdots$ & 23.7909 \\
\hline 63. & 14.7590 & 34.0616 & 33.6422 & - & $-\cdots$ & - \\
\hline 66. & 14.2585 & 32.9065 & 32.5013 & $--\cdot$ & -- & - \\
\hline 69. & 13.7750 & 31.7906 & 31.3992 & - & $\ldots$ & - \\
\hline 72. & 13.3079 & 30.7125 & 30.3345 & 20.7185 & -19.4101 & 20.7185 \\
\hline 75. & 12.8566 & 29.6710 & 29.3058 & $-\cdot$ & - & --- \\
\hline 78. & 12.4206 & 28.6648 & 28.3120 & - & 一 & -- \\
\hline 81. & 11.9994 & 27.6928 & 27.3519 & $\ldots$ & - & -- \\
\hline 84. & 11.5925 & 26.7537 & 26.4243 & 18.0477 & - & 18.0477 \\
\hline 87. & 11.1993 & 25.8464 & 25.5281 & $-\ldots$ & - . & -- \\
\hline 90. & 10.8196 & 24.9900 & 24.6625 & $\ldots$ & - & $\cdots$ \\
\hline 93. & 10.4527 & 24.1231 & 23.8262 & -- & - & - \\
\hline 96. & 10.0982 & 23.3051 & 23.0182 & 15.1212 & -14.7295 & 15.7212 \\
\hline
\end{tabular}

${ }^{a}$ Used equations for two-compartment open model with first-order absorption with $k_{12}=1.162 . k_{21}=0.515, k_{\mathrm{e}}=0.038$, and $k_{a}=0.5 \mathrm{hr}^{-1}, C_{0}=100$. These parameters gave equations

$$
\begin{aligned}
& C_{1}=30.458 e^{-0.0115 t}-29.1825 e^{-1.7035 t}-1.2757 e^{-0.5 t} \\
& C_{2}=70.2929 e^{-0.0115 t}+28.5318 e^{-1.0735 t}-98.8247 e^{-0.5 t}
\end{aligned}
$$


2) a similar dramatic effect on $\hat{C}_{2}$ is not seen. Of course, there is a lower limit, and one must take sufficient samples to get a good distribution of points at least up to the peak of the $C_{1}, t$ curve (which in this example is near $4 \mathrm{hr}$ ). Notice that although set 3 consists of 15 samples and set 4 consists of only 14 samples the $C_{2}$ values are better in set 4 than in set 3 . The large $\Delta t$ of $24 \mathrm{hr}$ (from 24 to $48 \mathrm{hr}$ ) in set 3 caused $\hat{C}_{2}$ to become a large negative value at $48 \mathrm{hr}$.

$A_{t_{n}} / V_{1}$ values for each set were estimated by applying equation 1, the trapezoidal rule for the areas, and the $\hat{C}_{2}$ values listed in Table III. Then the $A_{t_{n}} V_{1}, t_{n}$ values were analyzed by two different methods to estimate the $A_{x} / V_{1}$ and $k_{a}$ values. The Guggenheim method was applied to the equally spaced values in the 0 - to 2 -hr range. Since absorption had essentially ceased at $10 \mathrm{hr}$ all the $A_{i_{n}} / V_{1}$ values from 12 to $96 \mathrm{hr}$ were averaged to obtain an estimate of the asymptote of each set: the early $A_{t_{n}} / V_{1}$ values were then subtracted from these asymptotes and the natural logarithms of the differences were treated by least squares to obtain the estimate of $k_{a}$ by the usual sigma-minus method. Results are shown in Table IV. In each case, the Guggenheim method very accurately estimated both $A_{\alpha} / V_{1}$ and $k_{a}$. However, only for set 1 did the sigma-minus method give good estimates of either $k_{a}$ or $A_{\infty} / V_{1}$. The sigma-minus plots are shown in Fig. 3. The unwary (with real data) might interpret data set 2 as being a case of biexponential absorption! The reason for these results is that the poor estimates of $C_{2}$ beyond $12 \mathrm{hr}$ in sets $2-4$ caused great fluctuation in the terminal $A_{t_{n} / V_{1}}$ values and all the values were lower than the real values.

\section{DISCUSSION}

The derivations have shown that under certain conditions the LooRiegelman method will provide the correct $A_{x} / V_{1}$ value and the correct

Table IV. How Both the Values of $\Delta C_{1}$ and $\Delta t$ (Data Set) and the Method of Plotting $A_{T} V_{1}$ Data Affect the Estimates of $A_{x} V_{1}$ and $k_{a}$ Obtained (Simulation Example 3)

\begin{tabular}{ccccc}
\hline \multirow{2}{*}{$\begin{array}{c}\text { Data } \\
\text { set }\end{array}$} & \multicolumn{2}{c}{ Parameter } & \multicolumn{2}{c}{ Estimate obtained from $A_{T} / V_{\mathrm{t}}$ data by } \\
\cline { 2 - 5 } & Symbol & Real value & Guggenheim method & Sigma-minus plot \\
\hline \multirow{2}{*}{1} & $A_{x} / V_{1}$ & 100.0 & 100.1 & 99.6 \\
& $k_{a}$ & 0.50 & 0.4988 & 0.5164 \\
2 & $A_{x} / V_{1}$ & 100.0 & 100.1 & 90.2 \\
& $k_{a}$ & 0.50 & 0.4988 & 0.5887 \\
3 & $A_{x} / V_{1}$ & 100.0 & 100.5 & \\
& $k_{a}$ & 0.50 & 0.4941 & Curved plot \\
4 & $A_{x} / V_{\mathrm{t}}$ & 100.0 & 101.1 & 98.1 \\
& $k_{a}$ & 0.50 & 0.4863 & 0.6138 \\
\hline
\end{tabular}




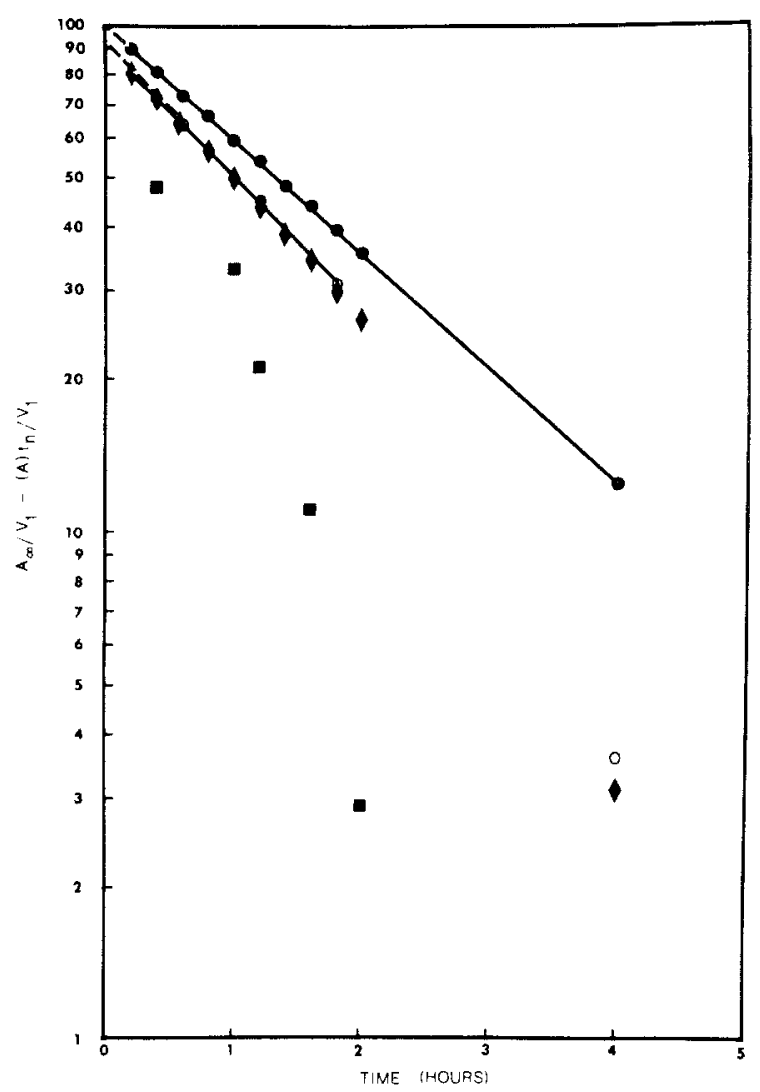

Fig. 3. Sigma-minus plots for simulation example 3. - Set 1 ; $\bullet$, set $2 ; \mathbf{\square}$, set $3 ; O$, set 4 .

kinetics of absorption (or the correct $A_{T} / V_{1}, T$ plot if the kinetics of absorption are nonuniform) independently of whether there is metabolism in compartment 2 or not. In those cases where metabolism does occur in compartment 2, the disposition parameters estimated from the intravenous data are only apparent and not the real values. The relationships among the parameters of Models I, II, and III are shown. This explains the results reported by Kaplan (5) and Breckenridge and Orme (6). The treatment herein is simpler than that of Suzuki and Saitoh (4).

In situations where the disposition parameters are biased, such as when they are obtained by the "feathering" or "back-projection" technique or when the computer converges on a local minimum in the least-squares surface, use of such biased parameters in application of the Loo-Riegelman 
method to oral data results in reasonable estimate of the true absorption rate, $k_{a}$ (if absorption is first order), but in a biased value of $\hat{A_{\infty} / V_{1}}$ which is approximately given by equation 44 . This strongly suggests that in the fitting of the intravenous concentration data one should use as good initial estimates of the parameters $k_{12}, k_{21}, k_{\mathrm{el}}$, and $C_{0}$ for Model II as possible, and fit the data to equation 41 repeatedly on the computer until one has the best curve-fit possible-that is, there should be no systemic deviations of the model-predicted $\hat{C}_{1}$ from the observed $C_{1}$ values.

The reason there is bias when one "feathers" or "strips" the intravenous data is that to obtain the estimate of $\beta$ one assumes $e^{-\alpha t}=0$ at some point and then for terminal concentration data assumes $\beta=-\Delta \ln C / \Delta t$. This biased estimate of $\beta$ is higher than the true value, hence the residuals are biased, and a biased estimate of $\alpha$ is also obtained. The amount of bias is very dependent on the particular set of data which is "stripped." Besides the usual definition of $\beta$, one may define it as

$$
[d \ln C / d t]_{\substack{t \rightarrow x \\ C \rightarrow 0}}=-\beta
$$

When the digital computer obtains a fit without systematic deviations, it is really using this latter interpretation of $\beta$ rather than the former.

The Loo-Riegelman method assumes that the plasma level curve is linear between adjacent points. Because of this assumption, the values of $C_{2}$ estimated by the method depend on the time intervals between samples. Hence, optionally, in applying the method to oral concentration data one should have a large number of concentration-time points which are closely spaced. But this is impractical since one can take only so many blood samples following any given treatment in a human subject. To circumvent the problem, one can fit a function or functions to the data points such that the "line" goes through each observed concentration and is "smooth" between observed concentrations. An ideal approach, which the author has studied, is use of the spline and Akima methods reported by Fried and Zeitz (9). With a very "steep-slope" plasma concentration curve (sum of four exponential terms), Sedman (personal communication) has shown that the method gives interpolated concentrations between adjacent "observed" points which differ only $0-2 \%$ from the real values. Hence in applying the Loo-Riegelman method to oral concentration data one should generate a large number of such interpolated points and use these and the observed concentrations to estimate the $C_{2}$ values. We now have a computer program to interpolate such values and apply equations 1 and 2 to the interpolated and observed values. ${ }^{2}$

${ }^{2}$ P. Larson and A. Sedman, University of Michigan, Ann Arbor, Mich. 
In most simulations, the $A_{x} / V_{1}$ value, and sometimes the $k_{a}$ value, is better estimated by means of the Guggenheim method from the early $A_{T} / V_{1}$ values than by the classical sigma-minus method. The Guggenheim method requires equally spaced $A_{T} / V_{1}$ values and hence equally spaced blood samples during the absorption phase. When using the curve-fitting method above, one can generate interpolated concentrations at equally spaced time values, and hence the blood samples need not actually be taken at equally spaced intervals to apply the Guggenheim method. However, if the operator wishes to insure that he used all observed concentrations in the absorption phase, then to apply the Guggenheim method the study should be designed for taking blood samples at equally spaced intervals at least up to the time of the expected maximum plasma concentration.

If absorption is not first order, then both the Guggenheim plot of $\log \left(\Delta A_{t_{n}} / V_{1}\right)$ vs. $t_{1}$ and the sigma-minus plot will be curved if the data give a sufficient span of time compared with the time for half of the drug to be absorbed. In these cases, the user usually likes to present the $A_{t_{n}} / V_{1}$ vs. $t$ plot. If only the observed concentration data are used, then the problem presented by simulation example 3 (Table III) may occur and the $A_{x} / V_{1}$ value may be difficult to determine. However, if one uses a large number of interpolated values, then an estimate close to the true value of $A_{\infty} / V_{1}$ would be obtained: this is supported by set 1 in Table III. The relative $A_{x} / V_{1}$ values from different treatments are important in bioavailability studies and it is desirable to obtain the best possible estimates of this parameter.

\section{ACKNOWLEDGMENT}

The author is grateful to Dr. Carl Metzler, The Upjohn Company, Kalamazoo, Michigan, for generating the data discussed in simulation example 2 and for fitting these data with the program NONLIN.

\section{REFERENCES}

1. J. C. K. Loo and S. Riegelman. A new method for calculating intrinsic absorption rates of drugs. J. Pharm. Sci. 57: 918-928 (1968).

2. J. C. K. Loo and S. Riegelman. Some alternate methods for calculating the intrinsic absorption rate of drugs. Presented at the A.Ph.A. Academy of Pharmaceutical Sciences meeting. San Francisco, March 25-April 2, 1971.

3. L. W. Dittert. W. A. Cressman, S. A. Kaplan, S. Riegelman, and J. G. Wagner. Guidelines for Biopharmaceutical Studies in Man. A.Ph.A. Academy of Pharmaceutical Sciences, Washington, D.C., 1972, p. 14.

4. T. Suzuki and Y. Saitoh. Pharmacokinetic analysis of blood level data interpreted by a two compartment model. Chem. Pharm. Bull. 21: 1458-1469 (1973).

5. S. A. Kaplan. Pharmacokinetic profile of coumermycin $A_{1}$. J. Pharm. Sci. 59: 309-313 (1970). 
6. A. Breckenridge and M. Orme. Kinetics of warfarin absorption in man. Clin. Pharmacol. Ther. 14: 955-961 (1973).

7. J. G. Wagner. Use of computers in pharmacokinetics. Clin. Pharmacol. Ther. 8: 201-218 (1967).

8. J. G. Wagner and C. M. Metzler. Estimation of rate constants for absorption and elimination from blcud concentration data. J. Pharm. Sci. 56: 658-659 (1967).

9. J. Fried and S. Zeitz. Curve fitting by spline and Akima methods: Possibility of interpolation error and its suppression. Phys. Med. Biol. 18: 550-558 (1973). 